\title{
Effect of cancer-associated mutations in the PlexinB1 gene
}

Chun Zhou ${ }^{1,2 \dagger}$, Oscar Gee-Wan Wong ${ }^{1,3+}$, John R Masters ${ }^{1}$ and Magali Williamson ${ }^{1 *}$

\begin{abstract}
Background: Semaphorins act as chemotactic cues for cell movement via their transmembrane receptors, plexins. Somatic missense mutations in the plexinB1 gene coupled with overexpression of the protein frequently occur in prostate tumours, indicating a role for plexinB1 in the pathogenesis of prostate cancer.

Results: Two specific mutations found in prostate cancer enhance RhoD binding and one other mutation results in loss of inhibition of Rac-dependent Pak1 phosphorylation and lamellipodia formation and in impairment of trafficking of plexinB1 to the membrane. None of the three characterised mutations affect PDZRhoGEF binding, RhoA activity, the interaction of plexinB1 with the oncogenes ErbB2 or c-Met or ErbB2 phosphorylation. The mutations have the net effect of increasing cell motility by blocking plexinB1-mediated inhibition of Rac while enhancing the interaction with RhoD, an anti-migratory factor.

Conclusions: PlexinB1 mutations block plexinB1-mediated signalling pathways that inhibit cell motility.

Keywords: Plexin, Prostate cancer, Semaphorin, Rac, RhoD, ErbB2, c-Met
\end{abstract}

\section{Background}

Semaphorins are a group of 20 or more secreted or membrane bound proteins [1] that act as chemotactic cues for cells expressing their transmembrane receptors plexins [2]. Semaphorins affect cell behaviour in diverse ways, regulating cell motility [3], invasive capacity [4], adhesion [5] and cell and axon growth cone collapse [6]. Semaphorins consequently have a function in many physiological processes including angiogenesis [7], cell migration $[8,9]$, immune regulation [10] and organogenesis, affecting nervous system [11,12], lung [13], kidney [14] and cardiovascular development $[15,16]$ and epithelial-mesenchymal interactions [14]. The response of a cell to semaphorin stimulation depends on the type of responding cell and particular semaphorins can generate opposite reactions depending on cell type [17]. The transmembrane semaphorin receptors, plexins, either bind semaphorins directly, or in the case of most class 3 semaphorins, to a complex of neuropilins and plexins

\footnotetext{
* Correspondence: magali.williamson@ucl.ac.uk

† Contributed equally

'Prostate Cancer Research Centre, University College London, 67, Riding

House Street, London W1W 7EJ, UK

Full list of author information is available at the end of the article
}

$[2,18]$. Semaphorin 4D (Sema4D) binds directly to its receptor, plexinB1 [2].

Semaphorin/plexin signalling results in activation of receptor tyrosine kinases and modulation of the actin cytoskeleton via regulation of several specific small RhoGTPases. PlexinB1 binds to RacGTP $[19,20]$, sequestering it from its downstream effectors, such as Pak1 [21], and to Rnd [22] and RhoD [23]. Rac, Rnd and RhoD all bind to the same region in the cytoplasmic domain of plexinB1, the RhoGTPase binding domain (RBD) [23]. Rnd binding is required for the binding of R-Ras to plexinB1 and for the R-RasGTPase activating protein (GAP) activity of plexinB1 which inactivates R-Ras resulting in a decrease in integrin and PI3K activation $[24,25]$. Sema4D/plexinB1 signalling activates RhoA through activation of PDZRhoGEF and LARG which bind to the Cterminal of the plexinB1 protein [26]. PlexinB1 can also mediate the inhibition of RhoA via the recruitment of p190RhoGAP [27]. PlexinB1 interacts with the receptor tyrosine kinases c-Met [28] and ErbB2 [29] via their extracellular domains and Sema4D/plexinB1 signalling results in c-Met and ErbB2 phosphorylation [28,29]. Activated ErbB2 phosphorylates tyrosines on plexinB1 creating a binding site for PLC $\gamma$. PLC $\gamma$ binding is required for Sema4D-mediated RhoA activation [30]. Sema4D can

\section{() Biomed Central}


both promote and inhibit migration depending on the plexinB1 co-receptors expressed [31].

Crystal structures of the RBD [23] and of the cytoplasmic domain of plexinB1 in complex with Rac1 $[32,33]$ have been determined. Bell et al. [33]., identified a second RhoGTPase binding site in addition to the RBD, adjacent to the Ras site, which stabilises a trimeric structure of plexinB1-Rac complexes.

We have previously found mutations in the plexinB1 gene in $8 / 9$ prostate cancer bone metastases, $7 / 17$ prostate cancer lymph node metastases and $41 / 89$ primary cancers, together with overexpression of the protein [34]. The mutations in plexinB1 enhance adhesion, migration and invasion in vitro and inhibit cell collapse [34]. The finding of functionally significant mutations in plexinB1 in prostate tumours and overexpression of the plexinB1 protein suggests that plexinB1 has a role in prostate cancer and so is a potential target for therapy. However, the mechanism by which plexinB1 contributes to prostate cancer progression is yet to be determined.

\section{Results}

\section{Mutations in plexinB1 increase RhoD binding}

RhoD binds to the RBD domain of plexinB1 in the region where mutations are found in prostate cancer [35]. To determine if the mutations affect binding of RhoD to plexinB1 we performed GST pull down assays using GSTcyto-plexinB1(wild type (WT) or mutant) fusion proteins and dominant negative (T31K) or constitutively active (G26V) RhoD. Three mutations were characterised in these experiments: A5359G (amino acid change T1697A), A5653G (amino acid change T1795A) and T5714C (amino acid change L1815P). Constitutively active RhoD (G26V) bound to the cytoplasmic domain of WT plexinB1, but dominant negative RhoD(T31K) did not (Figure 1). Binding of RhoDGTP to plexinB1 was not inhibited by the mutations in plexinB1. The interaction was enhanced by the presence of mutations A5359G (T1697A) and A5653G (T1795A) in the cytoplasmic domain of plexinB1 (Figure 1). To test the effect of RhoDGTP expression on plexinB1 function, we performed collapse assays on COS7 cells co-transfected with plexinB1, Rnd, and RhoD (G26V) or RhoD(T31K). Expression of constitutively active RhoD significantly reduced cell collapse (Figure $1 C$ ).

\section{Mutation of plexinB1 results in loss of inhibition of RacL61-dependent Pak1 phosphorylation}

PlexinB1 on the plasma membrane binds to and sequesters Rac-GTP and inhibits it from activating downstream effectors such as p21-activated kinase1 (Pak1) [21]. We have previously found that binding of Rac1 to the cytoplasmic domain of plexinB1 is inhibited by L1815P and is diminished by T1795A and T1697A amino acid changes [34]. To test the effect of cancer-

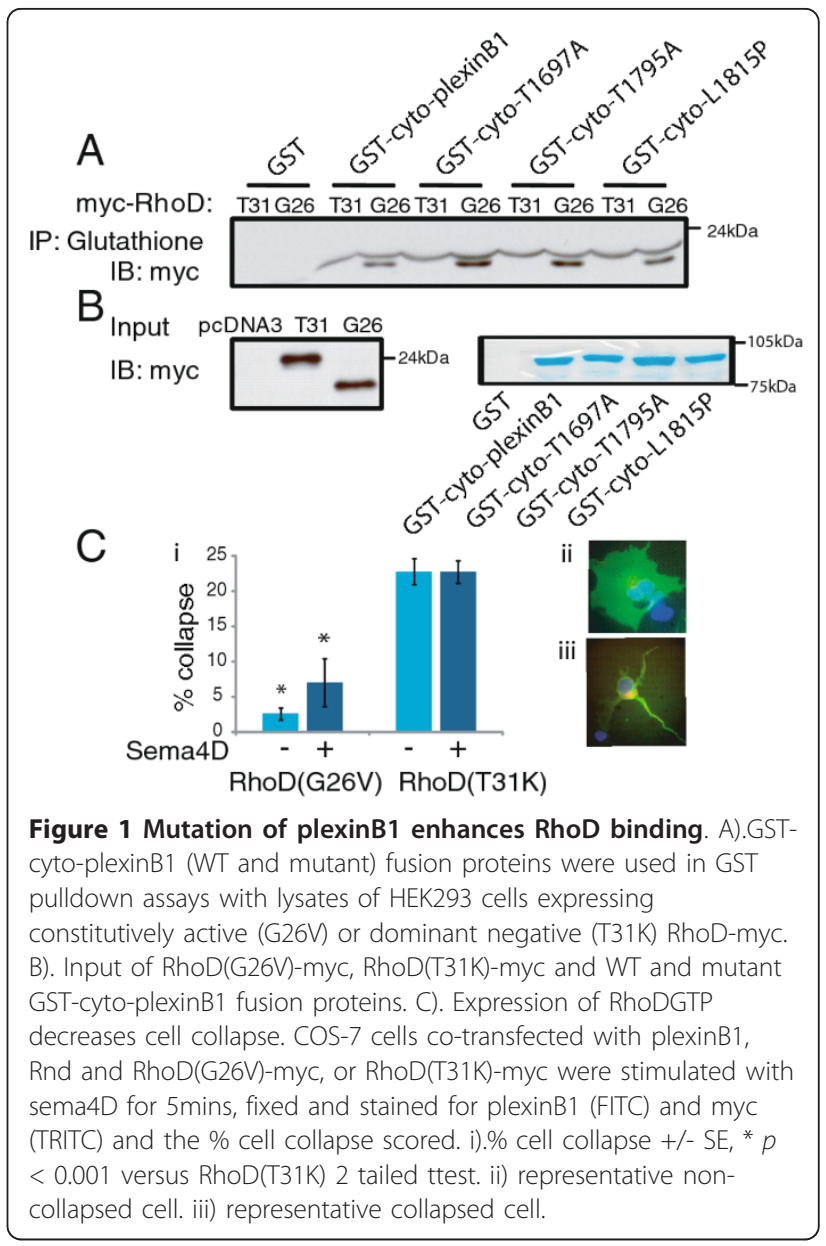

associated mutations of plexinB1 on Rac-dependent Pak1 phosphorylation, WT plexinB1 or three different mutant forms of the protein were co-expressed with RacL61 and Pak1 in COS-7 cells. The phosphorylation status of Pak1 was monitored by western blotting using anti-phospho199/204-Pak1 antibody. As previously reported [36] coexpression of Rac1L61 and Pak1 stimulated the autophosphorylation of Pak1 (Figure 2A). Coexpression of WT plexinB1 with Rac1L61 and Pak1 inhibited Pak1phosphorylation. In contrast co-expression of plexinB1 (T1795A) or plexinB1 (L1815P) with Rac1L61 and Pak1 did not inhibit Pak1 phosphorylation. PlexinB1 (T1697A) inhibited Rac-dependent Pak1 phosphorylation to a similar extent as WT. Therefore the T5714C(L1815P) and A5653G (T1795A) mutations in plexinB1 result in loss of plexinB1-mediated inhibition of Rac-dependent Pak1 phosphorylation.

\section{Mutation of plexinB1 results in loss of inhibition of RacL61-dependent cell spreading}

Cell spreading and extension of lamellipodia are phenotypes characteristic of Rac1 activation. COS-7 cells 


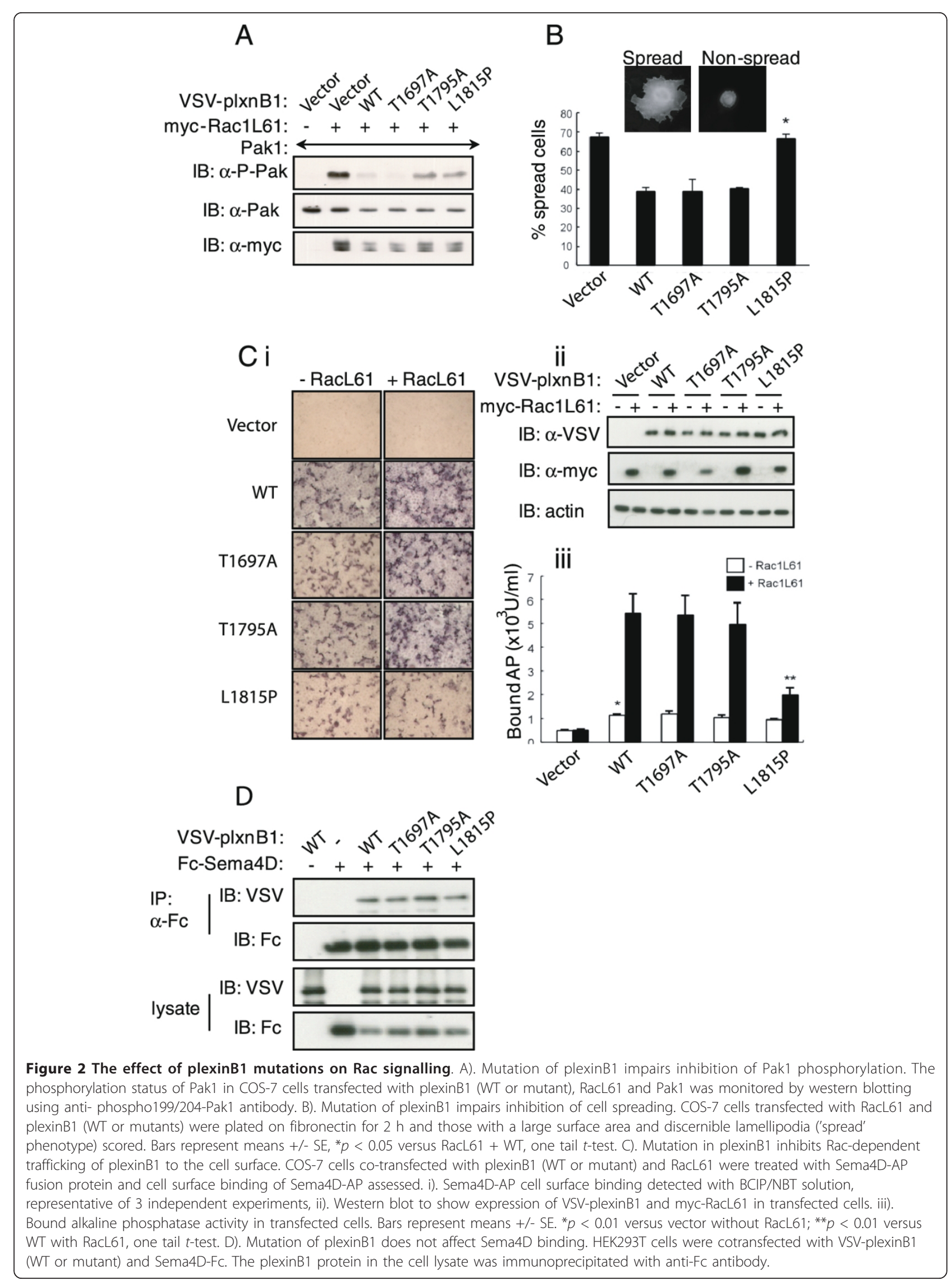


transfected with RacL61 exhibit a "spread" phenotype with a large surface area and discernible lamellipodia 2 $\mathrm{h}$ after plating on fibronectin (Figure 2B). Expression of WT plexinB1 reduced the number of cells showing this phenotype, consistent with the binding and sequestration of RacGTP by plexinB1, which effectively prevents Rac1 from participating in other signaling pathways. The inhibitory effect on cell spreading was retained in cells expressing plexinB1 (T1697A) and (T1795A). In contrast, the inhibitory effect on cell spreading was lost in cells expressing plexinB1 (L1815P).

\section{Mutation of plexinB1 inhibits Rac-dependent trafficking of plexinB1 to the cell surface}

Rac1-GTP binds to and facilitates the cell surface expression of plexinB1 [21]. We sought to determine if mutation of plexinB1 affects Rac-dependent cell surface trafficking of plexinB1.

COS-7 cells co-transfected with plexinB1 (WT or mutant) and RacL61 were treated with Sema4D-AP fusion protein and cell surface binding of Sema4D-AP assessed. In the absence of RacL61 co-expression, COS7 cells expressing WT or three mutant forms of plexinB1 showed similar levels of Sema4D-AP cell surface binding, significantly higher than cells not transfected with plexinB1 $(p<0.01)$ (Figure 2 C). Sema4D-AP cell surface binding was enhanced when RacL61 was coexpressed with WT plexinB1. Similarly, cell surface binding of Sema4D-AP to COS-7 cells was enhanced when RacL61 was co-expressed with plexinB1(T1697A) and plexinB1(T1795A) to a similar extent as WT. In contrast, co-expression of RacL61 with plexinB1 (L1815P), did not enhance cell surface binding of Sema4D-AP. A significant difference in staining intensity was observed between cells expressing WT and plexinB1 (L1815P) in the presence of Rac1L61 $(p<0.01)$ but not in the absence of Rac1L61. As the Sema4D binding capacity of plexinB1 was not affected by the mutations (as shown by immunoprecipitation, Figure 2D), our results show that the L1815P change inhibits Racdependent trafficking of plexinB1 to the cell surface.

Taken together, these results show that the T5714C (L1815P) mutation renders plexinB1 defective in sequestering and inhibiting Rac1 function and in facilitating plexinB1 trafficking. The A5653G (T1795A) mutation, which reduces RacGTP binding, also failed to inhibit Pak1 activation, although no discernible effect on cell spread or trafficking was seen.

\section{Effect of mutations on RhoA activation}

PlexinB1 binds to the RhoGEFs, PDZRhoGEF and LARG, resulting in activation of RhoA. We investigated whether the mutations affect the interaction between PDZRhoGEF and plexinB1 and plexinB1-mediated Rho activation.
Immunoprecipitation of PDZRhoGEF with anti-VSV from lysates of HEK293 cells co-expressing PDZRhoGEF and VSV-plexinB1 indicated that the mutations have little effect on PDZRhoGEF binding to plexinB1 (Figure 3A). Rho activation assays, using rhotekin pull down which binds to active RhoGTP selectively, showed that mutant forms of plexinB1 activate Rho to a similar extent as the WT form of plexinB1(Figure 3B). Expression of exogenous plexinB1 (WT or mutant) resulted in Rho activation in both the presence and absence of Sema4D, showing that overexpression of the plexinB1 receptor can mimic ligand binding as shown before [28]. Therefore the mutations do not affect plexinB1-mediated Rho activation.

PlexinB1 can also inhibit Rho activation via its interaction with p190RhoGAP [27]. Expression of WT plexinB1 inhibits the formation of stress fibres (an indicator of Rho activity) in COS-7 cells (Figure 3C), suggesting that RhoA is inhibited by plexinB1 in these cells, possibly via p190RhoGEF. The ability of three plexinB1 mutants to inhibit stress fibre formation was not significantly different from that of WT (Figure 3D). Taken together we saw no evidence that the three mutant forms of plexinB1 tested affect RhoA signalling.

Effect of mutations on the interaction of ErbB2 and c-met with plexinB1 and on plexinB1-mediated phosphorylation of ErbB2

PlexinB1 interacts with and activates the receptor tyrosine kinases ErbB2 [29] and c-Met [28] and Sema4D stimulation results in phosphorylation of both proteins in transfected HEK293 cells [31]. To determine if mutations of plexinB1 affect the interaction of plexinB1 with ErbB2, HEK293 cells were co-transfected with VSV-plexinB1 (WT or mutant) and ErbB2. ErbB2 was co- immunoprecipitated with anti-VSV (Figure 4A). Both WT and mutant forms of plexinB1 interacted with ErbB2 and the strength of interaction between the different mutant forms of plexinB1 and the WT form were comparable. Similarly, all mutant forms of plexinB1 tested bound to c-Met to an equivalent extent as WT plexinB1 in cells co-expressing VSV-plexinB1 (WT or mutant) and c-Met (Figure 4B). WT and mutant forms of plexinB1 pulled down the $170 \mathrm{kDa}$ extracellular form of $\mathrm{c}$-Met indicating that different forms of plexinB1 interact with the extracellular domain of c-Met. Therefore mutations in plexinB1 do not affect the interaction of plexinB1 with ErbB2 or c-Met. Exogenous expression of WT plexinB1 increased phosphorylation of ErbB2 (Figure 4C), and the mutations had little effect on this process.

\section{Discussion}

Mutations in the gene for plexinB1 are frequent in prostate cancer and the plexinB1 protein is overexpressed in prostate tumours, indicating that plexinB1 has a role in 


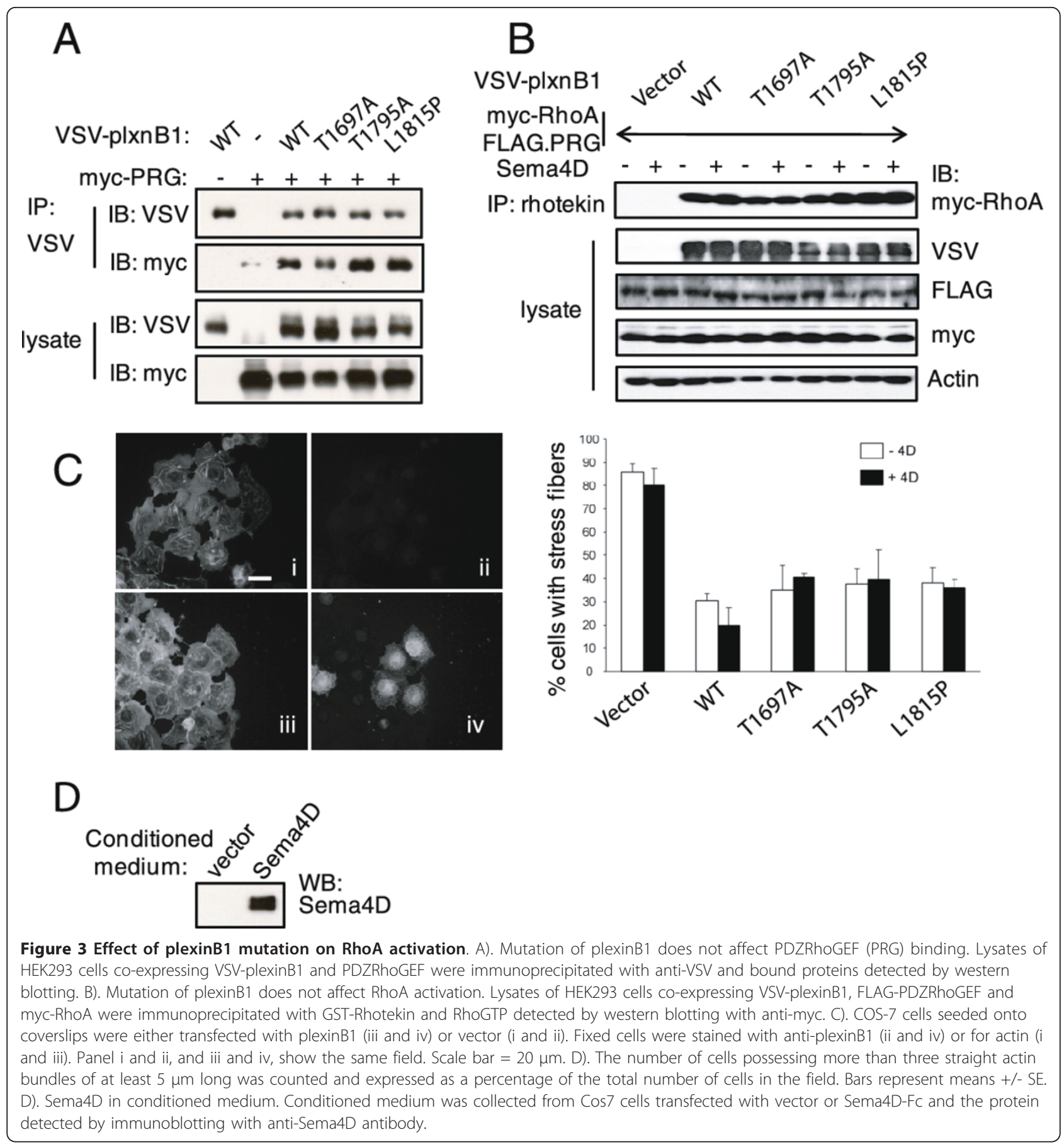

prostate cancer. The aim of this study was to investigate the mechanism by which mutation of plexinB1 contributes to prostate cancer progression. The three different cancer-associated plexinB1 mutations that were investigated in this study affect plexinB1-small RhoGTPase signalling in different ways, but have no effect on ErbB2, cMet binding or RhoA activity.

Surprisingly, in spite of its position in the RBD region of plexinB1, the L1815P change does not impair RhoD binding. Rac has been shown to bind to a second site close to the R-Ras binding site in the cytoplasmic domain of plexinB1, in addition to the RBD site [33]. It is possible that RhoD also binds to this second site (site $B$ in reference [33]) so that mutations in the RBD would not prevent RhoD binding. Alternatively the mode of binding of RhoD to the RBD may be different from that of the Rnd1 and Rac1. In an analogous way, the L1815P change inhibits Rnd1 binding to the RBD but not Rnd2 


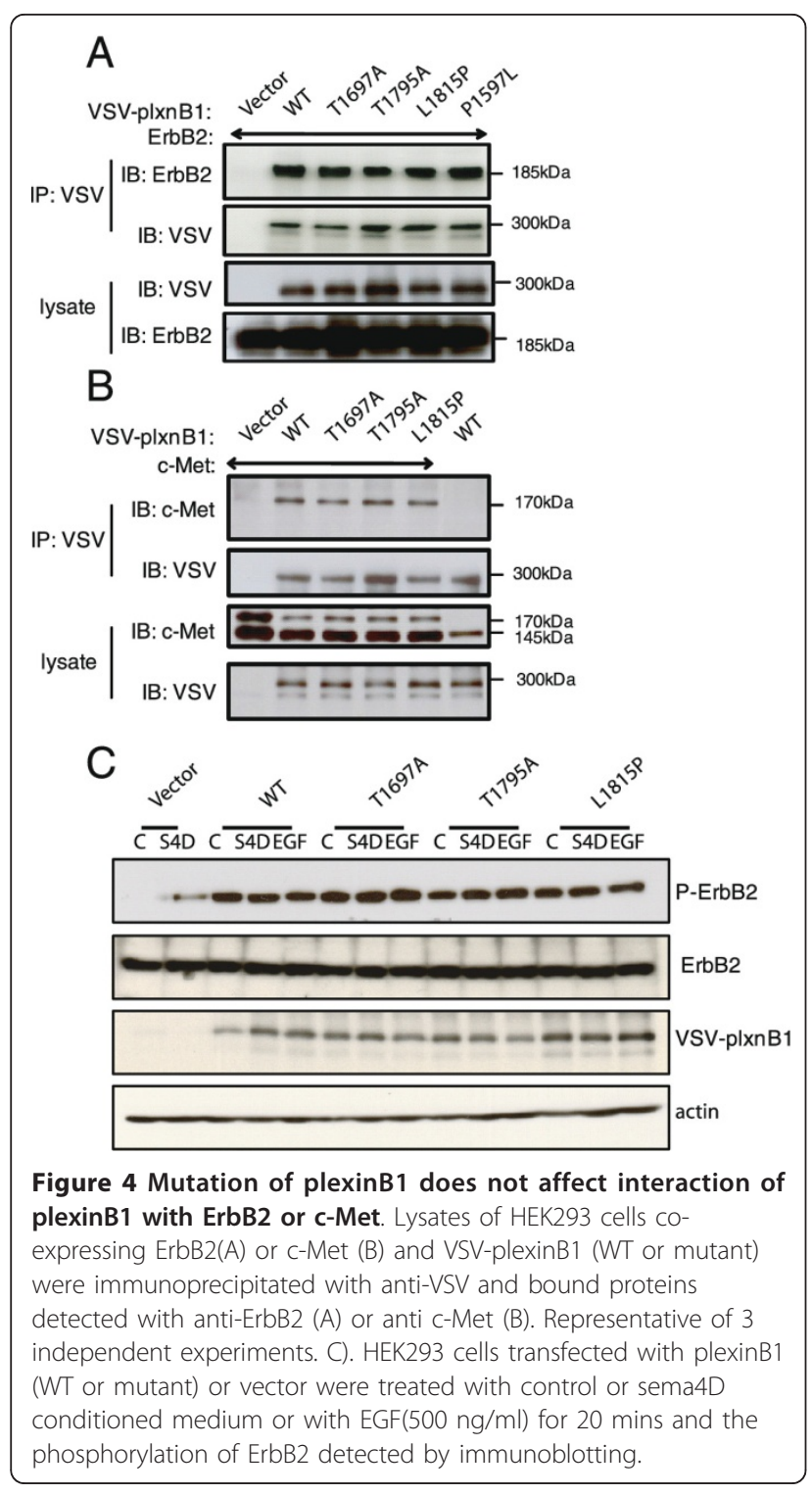

binding [37]. Binding of RhoDGTP to plexinB1 is also retained by the T1795A and T1697A mutant forms, and binding is enhanced by these mutations. The T1795A amino acid change occurs within the RBD region and the T1697A change occurs outside of this region [35].

All 3 mutant forms have lost R-RasGAP activity, yet only the L1815P mutation inhibits Rnd binding. Loss of Rnd1 binding would account for the loss of R-RasGAP activity in the L1815P mutant form, since R-RasGAP activity is dependent on Rnd binding [24]. In contrast, the T1795A and T1697A forms bind Rnd, yet R-RasGAP activity is lost. Interestingly, RhoD has been shown to antagonise Rnd in signalling via plexinA1 [38]. We have found that RhoDGTP reduced cell collapse when co-expressed with Rnd and plexinB1. If RhoD does antagonise Rnd signalling via plexinB1, the increase in
RhoD binding seen in the T1795A and T1697A mutant forms may contribute to the loss of R-Ras GAP activity seen for these forms.

RhoDGTP inhibits stress fibre formation and motility [39]. Binding of RhoDGTP to WT plexinB1 may sequester RhoD from downstream signalling partners. Expression of the T1697A and T1795A mutant forms of plexinB1 in tumour cells is predicted to have the effect of releasing the cell from the inhibitory effect of RhoD on motility and stress fibre formation, thereby promoting motility.

RacGTP binds and activates the autophosphorylation of Pak1 [36]. WT plexinB1 binds and sequesters RacGTP and thereby inhibits Pak1 phosphorylation [21]. Phosphorylation of Pak1 is not however inhibited by coexpression of RacGTP with the L1815P or T1795A mutant forms of plexinB1. The L1815P sequence change inhibits Rac, Rnd and R-Ras binding and the intrinsic RRasGAP activity of plexinB1 [34]. The T1795A mutation does not affect Rnd binding but reduces Rac binding and inhibits R-Ras binding and R-RasGAP activity [34]. The release of inhibition of Pak1 phosphorylation by tumour cells expressing the L1815P and T1795A mutant forms of plexinB1is expected to result in an increase in MAPK signalling and to promote tumour progression.

Activation of Rac results in reorganisation of the actin cytoskeleton to form lamellipodia at the leading edge of migrating cells, a phenotype that is inhibited by WT plexinB1 expression. The L1815P mutation in plexinB1 blocks this inhibition of lamellipodia formation. The release from lamellipodia inhibition is expected to result in an increase in motility, and is consistent with the increase in motility we have observed in cells expressing this mutant form of plexinB1 [34]. We have previously shown that overexpression of the three characterised mutations in HEK293 cells, without exogenous RacL61 expression, resulted in an increase in cell spreading above that of vector controls [34]. These results suggest that in addition to the loss of inhibition of Rac function shown here, the mutations confer a gain of function which results in an increase in cell spreading.

Rac has also been shown to act upstream of plexinB1 facilitating the trafficking of plexinB1 to the cell membrane. Cells expressing the L1815P mutated form of plexinB1 show a decrease in cell surface expression of the protein.

\section{Conclusions}

Activated plexinB1 functions either as a positive or negative regulator of several signalling pathways that promote cell migration. ErbB2, c-Met and RhoA are activated by plexinB1, enhancing cell migration. In contrast, Rac, Rnd and R-Ras are inhibited by plexinB1, resulting in a decrease in cell motility. The response of a cell to plexinB1 activation depends on a balance 
between these signaling pathways. The mutations we identified have no effect on ErbB2, c-Met or PDZRhoGEF binding or RhoA activity which enhance migration, but one or more of the mutations inhibit or hinder Rac, Rnd, and R-Ras binding and R-RasGAP [34] activity. The mutations thus have the net effect of increasing cell motility by the loss of inhibitory pathways. Two of the mutations also promote sequestration of RhoDGTP, an anti-migration factor.

\section{Methods}

\section{Plasmid constructs and cell culture}

The expression constructs for VSV-plexinB1and Sema4D-AP were kind gifts from Dr. L. Tamagnone. The region on plexinB1 cDNA encoding the intracellular domain (amino acids 1512-2135, accession no. X87904) was amplified by PCR and cloned into pGEX-4 T-3 (Amersham) using SalI and Xhol sites to produce pGEXB1cytoWT. The prostate cancer-associated mutations: L1815P, T1795A and T1697A were introduced into pGEXB1cytoWT and into VSV-plexinB1 by using QuikChange II XL in vitro mutagenesis kit (Stratagene). Other constructs were kindly provided by the following: Dr. KL Guan, pRK5-mycRacL61 and N17; Professor Takeshi Endo (Chiba University, Japan): RhoD pEFBOS/Myc-RhoD G26V and T31K; Dr. Hitoshi Kikutani (Osaka University, Japan): Sema4D-Fc; Dr. J. Swiercz (Heidelburg): FLAG-PDZRhoGEF, ErbB2, RhoA; Dr M. Driessens: myc-PDZRhoGEF; Prof. J.Chernoff (Fox Chase Cancer Centre): pCMV-myc-hPak1. HEK293 and COS-7 cells were grown in DMEM (10\% FCS).

\section{Antibodies}

The following antibodies were used: anti-VSV, anti-FLAG, anti-myc (Sigma, V4888, F7425, C956), anti-Human IgG Fc (Jackson ImmunoResearch, 109-005-098), anti- $\beta$-actin (Abcam, ab6276), anti-Pak1 (Chemicon, AB3844), anti-PPak, anti-phospho-ErbB2 (Cell Signalling), ErbB2 (Millipore), Sema4D and plexinB1 (ECM Biosciences), c-Met (c-28), plexinB1 (H300), (Santa Cruz).

\section{Rac-mediated Pak1 phosphorylation}

COS-7 cells were transfected with Pak1, constitutively active Rac (RacL61), and plexinB1(WT or mutant) or empty vector using Lipofectamine (Invitrogen). Lysates were analysed for Pak phosphorylation using a phosphoPak1 antibody.

\section{Recombinant Sema4D}

COS-7 cells transfected with Sema4D-Fc or Sema4D-AP or empty vector (control) were grown in serum free medium for $72 \mathrm{~h}$. The conditioned medium was collected and used directly or purified. Sema4D concentration was assessed by western blotting (Figure 3).

\section{Cell spread assays}

COS-7 cells transfected with myc-RacL61 and VSVplexinB1 (WT or mutant) or vector were plated on 10 $\mu \mathrm{g} / \mathrm{ml}$ fibronectin. Unattached cells were washed away after $2 \mathrm{~h}$. Attached cells were fixed (4\% paraformaldehyde), permeabilized ( $0.1 \%$ Triton-X 100$)$, blocked ( $2 \%$ BSA) and subjected to immunofluorescence staining (anti-myc). At least 200 myc-RacL61 positive cells were scored per slide.

\section{In situ binding of Sema4D-AP with plexinB1}

COS-7 cells transfected with plexinB1 (WT or mutant) and RacL61 or vector were washed with HBAH (Hanks balanced salt solution with $20 \mathrm{mM}$ HEPES pH 7.0, 0.5 $\mathrm{mg} / \mathrm{ml} \mathrm{BSA}, 0.1 \%(\mathrm{w} / \mathrm{v}) \mathrm{NaN} 3)$, treated with HBAH containing $1,000 \mathrm{ng} / \mathrm{ml}$ Sema4D-AP for $90 \mathrm{~min}$ then washed with ice-cold HBAH and fixed $(65 \%(\mathrm{v} / \mathrm{v})$ acetone, $8 \%(\mathrm{v} / \mathrm{v})$ formalin, $20 \mathrm{mM}$ HEPES $\mathrm{pH} 7.0)$. The cells were incubated at $65^{\circ} \mathrm{C}$ for $100 \mathrm{~min}$, then in $\mathrm{BCIP} /$ NBT solution in the dark.

\section{Immunoprecipitation}

Lysates of HEK293 transfected cells were incubated with $1 \mu \mathrm{g}$ of selective antibody for $2 \mathrm{~h}$ at $4^{\circ} \mathrm{C}$. The antigenantibody complex was incubated with Protein-G sepharose for $2 \mathrm{~h}$, washed 3 times and analyzed by immunoblotting.

\section{GST-pull down assay}

Lysates of COS-7 cells expressing RhoDG26V or RhoDT31K were incubated with GST-fused cyto-plexinB1 (WT or mutant) overnight. Following washing of the matrix, the samples were analysed by SDS-PAGE and immunoblotting.

\section{Collapse assay}

COS-7 cells plated on coverslips were co-transfected with plexinB1, Rnd and RhoDG26V-myc or RhoDT31Kmyc. $48 \mathrm{~h}$ post transfection, the cells were treated with sema4D $(4 \mu \mathrm{g} / \mathrm{ml})$ for 5 mins, then fixed with $4 \%$ paraformaldehyde, permeabilised with $0.2 \%$ triton, then stained by immunofluorescence using anti-plexinB1 antibody(R\&D) (FITC secondary antibody(Southern Biotech)) and anti-myc antibody (Abcam) (TRITC secondary). Co-transfected cells were sized using imageJ and cells with a size of $<500 \mu \mathrm{m} 2$ with 3 or more processes were scored as 'collapsed'. Cells were scored 'blind'. The experiment was performed $3 \times$ in triplicate with a total of 186 or more co-transfected cells counted per condition.

\section{Rho activity assay}

HEK293 cells were transfected with RhoA, PDZRhoGEF and plexinB1 (WT or mutant) or vector control. $48 \mathrm{~h}$ 
after transfection, cells were treated with control or Sema4D conditioned medium for 25 mins and the lysate incubated with $50 \mu \mathrm{l}$ Rhotekin (Upstate) for $1 \mathrm{~h}$, washed 3 times and the pulled down protein analysed by western blot with anti-myc antibody.

\section{Stress fibre assay}

COS-7 cells transfected with plexinB1 (WT or mutant) were fixed ( $4 \%$ paraformaldehyde), permeabilized $(0.1 \%$ Triton-X 100), blocked with $2 \%$ BSA then subjected to immunocytochemistry with anti-plexinB1 (SantaCruz) then goat-anti-mouse FITC (SouthernBiotech) supplemented with Phalloidin-TRITC (Sigma). The number of cells possessing more than three straight actin bundles of at least $5 \mu \mathrm{m}$ long was counted. The identities of the slides counted were blinded to the researcher. At least 50 cells were counted per slide.

\section{PlexinB1-mediated ErbB2 phosphorylation}

HEK293 cells transfected with vector or plexinB1 (WT or mutants) were serum starved over 2 nights then treated with control or sema4D conditioned medium or rhEGF(500 ng/ml, R\&D Biosytems) for 20 mins. Lysates were analysed for ErbB2 phosphorylation using an anti phospho-ErbB2 antibody.

\section{Acknowledgements}

We thank the Prostate Cancer Research Centre, Smith's Charity, the Rosetrees Trust, the Barcapel Foundation, the McAlpine Foundation and the Orchid Appeal for funding.

\section{Author details}

${ }^{1}$ Prostate Cancer Research Centre, University College London, 67, Riding House Street, London W1W 7EJ, UK. ${ }^{2}$ The Wistar Institute, 3601 Spruce Street, Philadelphia, PA 19104, USA. ${ }^{3}$ Department of Pathology, The University of Hong Kong, HKSAR, China.

\section{Authors' contributions}

$\mathrm{CZ}$ and $\mathrm{OW}$ carried out the molecular genetic studies. JM participated in its design and coordination. MW conceived of the study, coordinated it and drafted the manuscript. All authors read and approved the final manuscript.

\section{Competing interests}

A patent has been filed by M.W. and J.R.M. for mutations.

Received: 26 October 2011 Accepted: 9 March 2012

Published: 9 March 2012

\section{References}

1. Kolodkin AL, Matthes DJ, Goodman CS: The semaphorin genes encode a family of transmembrane and secreted growth cone guidance molecules. Cell 1993, 75:1389-1399.

2. Tamagnone L, Artigiani S, Chen H, He Z, Ming Gl, Song H, Chedotal A, Winberg ML, Goodman CS, Poo M, Tessier-Lavigne M, Comoglio PM: Plexins are a large family of receptors for transmembrane, secreted, and GPI-anchored semaphorins in vertebrates. Cell 1999, 99:71-80.

3. Tamagnone $L$, Comoglio PM: To move or not to move? EMBO Rep 2004, 5:356-361.

4. Trusolino L, Comoglio PM: Scatter-factor and semaphorin receptors: cell signalling for invasive growth. Nat Rev Cancer 2002, 2:289-300.

5. Serini G, Valdembri D, Zanivan S, Morterra G, Burkhardt C, Caccavari F, Zammataro L, Primo L, Tamagnone L, Logan M, Tessier-Lavigne M,
Taniguchi M, Puschel AW, Bussolino F: Class 3 semaphorins control vascular morphogenesis by inhibiting integrin function. Nature 2003, 424:391-397.

6. Luo Y, Raible D, Raper JA: Collapsin: a protein in brain that induces the collapse and paralysis of neuronal growth cones. Cell 1993, 75:217-227.

7. Neufeld G, Kessler O: The semaphorins: versatile regulators of tumour progression and tumour angiogenesis. Nat Rev Cancer 2008, 8:632-645.

8. Eickholt BJ, Mackenzie SL, Graham A, Walsh FS, Doherty P: Evidence for collapsin-1 functioning in the control of neural crest migration in both trunk and hindbrain regions. Development 1999, 126:2181-2189.

9. Tamamaki N, Fujimori K, Nojyo Y, Kaneko T, Takauji R: Evidence that Sema3A and Sema3F regulate the migration of GABAergic neurons in the developing neocortex. J Comp Neurol 2003, 455:238-248.

10. Kumanogoh A, Kikutani $\mathrm{H}$ : Roles of the semaphorin family in immune regulation. Adv Immunol 2003, 81:173-198.

11. Polleux F, Giger RJ, Ginty DD, Kolodkin AL, Ghosh A: Patterning of cortical efferent projections by semaphorin-neuropilin interactions. Science 1998, 282:1904-1906.

12. Marin O, Yaron A, Bagri A, Tessier-Lavigne M, Rubenstein JL: Sorting of striatal and cortical interneurons regulated by semaphorin-neuropilin interactions. Science 2001, 293:872-875.

13. Kagoshima $M$, Ito $T$ : Diverse gene expression and function of semaphorins in developing lung: positive and negative regulatory roles of semaphorins in lung branching morphogenesis. Genes Cells 2001, 6:559-571.

14. Korostylev A, Worzfeld T, Deng S, Friedel RH, Swiercz JM, Vodrazka P, Maier $V$, Hirschberg A, Ohoka Y, Inagaki S, Offermanns S, Kuner R: A functional role for semaphorin 4D/plexin B1 interactions in epithelial branching morphogenesis during organogenesis. Development 2008, 135:3333-3343.

15. Behar O, Golden JA, Mashimo H, Schoen FJ, Fishman MC: Semaphorin III is needed for normal patterning and growth of nerves, bones and heart. Nature 1996, 383:525-528.

16. Toyofuku T, Zhang H, Kumanogoh A, Takegahara N, Suto F, Kamei J, Aoki K, Yabuki M, Hori M, Fujisawa H, Kikutani H: Dual roles of Sema6D in cardiac morphogenesis through region-specific association of its receptor, Plexin-A1, with off-track and vascular endothelial growth factor receptor type 2. Genes Dev 2004, 18:435-447.

17. Chen $H, H e Z$, Tessier-Lavigne M: Axon guidance mechanisms: semaphorins as simultaneous repellents and anti-repellents. Nat Neurosci 1998, 1:436-439.

18. Chen H, He Z, Bagri A, Tessier-Lavigne M: Semaphorin-neuropilin interactions underlying sympathetic axon responses to class III semaphorins. Neuron 1998, 21:1283-1290.

19. Driessens $M H, H u H$, Nobes CD, Self A, Jordens I, Goodman CS, Hall A Plexin-B semaphorin receptors interact directly with active Rac and regulate the actin cytoskeleton by activating Rho. Curr Biol 2001, 11:339-344.

20. Vikis HG, Li W, He Z, Guan KL: The semaphorin receptor plexin-B1 specifically interacts with active Rac in a ligand-dependent manner. Proc Natl Acad Sci USA 2000, 97:12457-12462.

21. Vikis HG, Li W, Guan KL: The plexin-B1/Rac interaction inhibits PAK activation and enhances Sema4D ligand binding. Genes Dev 2002, 16:836-845.

22. Oinuma I, Katoh $H$, Harada A, Negishi M: Direct interaction of Rnd1 with Plexin-B1 regulates PDZ-RhoGEF-mediated Rho activation by Plexin-B1 and induces cell contraction in COS-7 cells. J Biol Chem 2003, 278:25671-25677.

23. Tong Y, Chugha P, Hota PK, Alviani RS, Li M, Tempel W, Shen L, Park HW, Buck M: Binding of Rac1, Rnd1, and RhoD to a novel Rho GTPase interaction motif destabilizes dimerization of the plexin-B1 effector domain. J Biol Chem 2007, 282:37215-37224

24. Oinuma I, Ishikawa $Y$, Katoh $H$, Negishi M: The Semaphorin 4D receptor Plexin-B1 is a GTPase activating protein for R-Ras. Science 2004, 305:862-865.

25. Oinuma I, Katoh H, Negishi M: Semaphorin 4D/Plexin-B1-mediated R-Ras GAP activity inhibits cell migration by regulating beta(1) integrin activity. J Cell Biol 2006, 173:601-613.

26. Swiercz JM, Kuner R, Behrens J, Offermanns S: Plexin-B1 directly interacts with PDZ-RhoGEF/LARG to regulate RhoA and growth cone morphology. Neuron 2002, 35:51-63. 
27. Barberis D, Casazza A, Sordella R, Corso S, Artigiani S, Settleman J, Comoglio PM, Tamagnone L: p190 Rho-GTPase activating protein associates with plexins and it is required for semaphorin signalling. I Cell Sci 2005, 118:4689-4700.

28. Giordano S, Corso S, Conrotto P, Artigiani S, Gilestro G, Barberis D, Tamagnone L, Comoglio PM: The semaphorin $4 \mathrm{D}$ receptor controls invasive growth by coupling with Met. Nat Cell Biol 2002, 4:720-724.

29. Swiercz JM, Kuner R, Offermanns S: Plexin-B1/RhoGEF-mediated RhoA activation involves the receptor tyrosine kinase ErbB-2. J Cell Biol 2004, 165:869-880.

30. Swiercz JM, Worzfeld T, Offermanns S: Semaphorin $4 D$ signaling requires the recruitment of phospholipase Cgamma into the plexin-B1 receptor complex. Mol Cell Biol 2009, 29:6321-6334.

31. Swiercz JM, Worzfeld T, Offermanns S: ErbB-2 and met reciprocally regulate cellular signaling via plexin-B1. J Biol Chem 2008, 283:1893-1901.

32. Tong Y, Hota PK, Penachioni JY, Hamaneh MB, Kim S, Alviani RS, Shen L, He H, Tempel W, Tamagnone L, Park HW, Buck M: Structure and function of the intracellular region of the plexin-b1 transmembrane receptor. J Biol Chem 2009, 284:35962-35972.

33. Bell $C H$, Aricescu AR, Jones EY, Siebold CA: Dual binding mode for RhoGTPases in plexin signalling. PLoS Biol 2011, 9:e1001134.

34. Wong OG, Nitkunan T, Oinuma I, Zhou C, Blanc V, Brown RS, Bott SR, Nariculam J, Box G, Munson P, Constantinou J, Feneley MR, Klocker H, Eccles SA, Negishi M, Freeman A, Masters JR, Williamson M: Plexin-B1 mutations in prostate cancer. Proc Natl Acad Sci USA 2007, 104:19040-19045.

35. Tong Y, Hota PK, Hamaneh MB, Buck M: Insights into oncogenic mutations of plexin-B1 based on the solution structure of the Rho GTPase binding domain. Structure 2008, 16:246-258.

36. Nikolic M, Chou MM, Lu W, Mayer BJ, Tsai LH: The p35/Cdk5 kinase is a neuron-specific Rac effector that inhibits Pak1 activity. Nature 1998, 395:194-198

37. Wang H, Hota PK, Tong Y, Li B, Shen L, Nedyalkova L, Borthakur S, Kim S, Tempel W, Buck M, Park HW: Structural basis of Rnd1 binding to plexin Rho GTPase binding domains (RBDs). J Biol Chem 2011, 286:26093-26106.

38. Zanata SM, Hovatta I, Rohm B, Puschel AW: Antagonistic effects of Rnd1 and RhoD GTPases regulate receptor activity in Semaphorin 3A-induced cytoskeletal collapse. J Neurosci 2002, 22:471-477.

39. Tsubakimoto K, Matsumoto K, Abe H, Ishii J, Amano M, Kaibuchi K, Endo T: Small GTPase RhoD suppresses cell migration and cytokinesis. Oncogene 1999, 18:2431-2440.

doi:10.1186/1476-4598-11-11

Cite this article as: Zhou et al: Effect of cancer-associated mutations in the PlexinB1 gene. Molecular Cancer 2012 11:11.

\section{Submit your next manuscript to BioMed Central and take full advantage of:}

- Convenient online submission

- Thorough peer review

- No space constraints or color figure charges

- Immediate publication on acceptance

- Inclusion in PubMed, CAS, Scopus and Google Scholar

- Research which is freely available for redistribution

Submit your manuscript at www.biomedcentral.com/submit
C Biomed Central 